\title{
THE GAUSSIAN FREE FIELD AND HADAMARD'S VARIATIONAL FORMULA
}

\author{
HAAKAN HEDENMALM AND PEKKA J. NIEMINEN
}

\begin{abstract}
Авsтract. We relate the Gaussian free field on a planar domain to the variational formula of Hadamard which explains the change of the Green function under a perturbation of the domain. This is accomplished by means of a natural integral operator - called the Hadamard operator associated with a given flow of growing domains.
\end{abstract}

\section{INTRODUCTION}

The Gaussian free field $\left(\mathrm{GFF}_{0}\right)$ on a planar domain $\Omega$ is an analogue of the classical Brownian motion with two temporal dimensions, which frequently appears as the continuum limit of discrete random processes and plays an important role in statistical physics (see [11] for a general survey). It can be defined, at least formally, as a random linear combination of an orthonormal basis $\psi_{j}(j=1,2,3, \ldots)$ of the Sobolev space $H_{0}^{1}(\Omega)$,

$$
\Psi=\sum_{j=1}^{+\infty} \xi_{j} \psi_{j},
$$

where $\xi_{j}(j=1,2,3, \ldots)$ is a sequence of independent standard real-valued Gaussian variables (i.e., taken from $\mathrm{N}(0,1)$ ). The space $H_{0}^{1}(\Omega)$ consists of real-valued functions and is equipped with the usual Dirichlet inner product. The resulting stochastic field $\Psi$ is conformally invariant with covariance structure determined by the Green function of the domain; thus the $\mathrm{GFF}_{0}$ also exhibits interesting connections to complex analysis and potential theory.

The purpose of this note is to relate the Gaussian free field to the classical variational formula due to Hadamard [5] which describes the change of the Green function under a perturbation of the boundary of the underlying domain. We interpret Hadamard's formula in terms of a natural and concrete integral operator - called by us the Hadamard operator - associated with a given flow of smooth domains. It supplies an isometric isomorphism $L^{2}(\Omega) \rightarrow H_{0}^{1}(\Omega)$, and, consequently, it can be used to produce the corresponding $\mathrm{GFF}_{0}$ field from the standard white noise field. This construction has certain appealing features since it enables us to view $\mathrm{GFF}_{0}$ as formed by adding independent infinitesimal harmonic fields whose boundary amplitudes correspond to suitably weighted white noise processes.

The paper is organized as follows. In Section 2 , we recall the definition of the space $H_{0}^{1}(\Omega)$ and some related prerequisites. In Section 3, we first describe a version of Hadamard's variational formula, and then proceed to introduce the associated Hadamard operator and analyse its properties. Section 4 contains a brief account on the white noise and Gaussian free fields from the point of view of Gaussian Hilbert spaces. Finally, in Section 5, we use the Hadamard operator to construct the Gaussian free field and derive some natural consequences.

Date: 8 February 2012.

2010 Mathematics Subject Classification. 60K35, 30C70.

Both authors were supported by the Göran Gustafsson Foundation (KVA) and Vetenskapsrådet (VR). The second author was in addition supported by the Academy of Finland, project 136785. 


\section{Preliminaries}

In this section, $\Omega$ is a finitely connected bounded domain in the complex plane $\mathbb{C}$ with a $C^{2}$-smooth boundary. We use the notation

$$
\Delta:=\frac{\partial^{2}}{\partial x^{2}}+\frac{\partial^{2}}{\partial y^{2}}, \quad \nabla:=\left(\frac{\partial}{\partial x}, \frac{\partial}{\partial y}\right), \quad \mathrm{d} A(z):=\mathrm{d} x \mathrm{~d} y,
$$

for the Laplacian, the gradient (nabla) operator, and the area element, respectively; here, $z=x+\mathrm{i} y$ is the decomposition of $z \in \mathbb{C}$ into real and imaginary parts.

2.1. The Green function and Poisson kernel. The Dirichlet Green function $G_{\Omega}$ in $\Omega$ solves, for $w \in \Omega$,

$$
\begin{cases}-\Delta_{z} G_{\Omega}(z, w)=\delta_{w}(z), & z \in \Omega, \\ G_{\Omega}(z, w)=0, & z \in \partial \Omega,\end{cases}
$$

where $\Delta_{z}$ indicates that the Laplacian is taken with respect to $z$. It has the symmetry property $G_{\Omega}(z, w)=G_{\Omega}(w, z)$. We write $\mathbf{G}_{\Omega}$ for the associated integral operator

$$
\mathbf{G}_{\Omega} f(z):=\int_{\Omega} G_{\Omega}(z, w) f(w) \mathrm{d} A(w), \quad z \in \Omega,
$$

extended to vanish in $\mathbb{C} \backslash \Omega$. Then, in the sense of distributions, $-\Delta \mathbf{G}_{\Omega} f=f$ in $\Omega$, so we may write $\mathbf{G}_{\Omega}=[-\Delta]^{-1}$.

The Poisson kernel $P_{\Omega}$ is obtained from

$$
P_{\Omega}(z, \zeta):=\frac{\partial}{\partial \mathrm{n}(\zeta)} G_{\Omega}(z, \zeta), \quad z \in \Omega, \zeta \in \partial \Omega,
$$

where the unit normal derivative is taken in the inward direction. For $\zeta \in \partial \Omega$, we extend $P_{\Omega}(z, \zeta)$ to (area-a.e.) $z \in \mathbb{C}$ by setting it equal to 0 in $\mathbb{C} \backslash \mathrm{cl}[\Omega]$, where cl[ $\left.\Omega\right]$ denotes the closure of $\Omega$. The associated integral operator is

$$
\mathbf{P}_{\Omega} f(z):=\int_{\partial \Omega} P_{\Omega}(z, \zeta) f(\zeta) \mathrm{d} s(\zeta), \quad z \in \Omega,
$$

where $\mathrm{d} s$ refers to the arc-length measure. It furnishes the harmonic extension of $f$ defined on $\partial \Omega$ to the interior $\Omega$.

2.2. The space $H_{0}^{1}(\Omega)$. The Sobolev space $H_{0}^{1}(\Omega)$, alternatively denoted by $W_{0}^{1,2}(\Omega)$, can be defined as the Hilbert space completion of (real-valued) $C_{0}^{\infty}(\Omega)$ under the Dirichlet inner product

$$
\langle f, g\rangle_{\Omega, \nabla}:=\int_{\Omega} \nabla f \cdot \nabla g \mathrm{~d} A,
$$

where the dot is the inner product in $\mathbb{R}^{2}$. The norm of the space is given by $\|f\|_{\Omega, \nabla}:=\langle f, f\rangle_{\Omega, \nabla}^{1 / 2}$. As all functions $f, g \in H_{0}^{1}(\Omega)$ vanish on the boundary, we may write, using integration by parts,

$$
\langle f, g\rangle_{\Omega, \nabla}=\langle-\Delta f, g\rangle_{\Omega}=\left\langle[-\Delta]^{1 / 2} f,[-\Delta]^{1 / 2} g\right\rangle_{\Omega},
$$

where $\langle\cdot, \cdot\rangle_{\Omega}$ is the usual inner product in $L^{2}(\Omega)$. It follows that the operator $[-\Delta]^{1 / 2}$ supplies an isometric isomorphism from $H_{0}^{1}(\Omega)$ onto $L^{2}(\Omega)$, and its inverse is given by $[-\Delta]^{-1 / 2}$. As is standard, the powers of $-\Delta$ are understood in terms of eigenfunction expansions with vanishing Dirichlet boundary data (cf., e.g., [3, Sec. 6.5.1]). 


\section{HAdAmARD's Formula AND AN ASSOCiATED OPERATOR}

3.1. Hadamard's variational formula. We consider a family of bounded planar domains $\Omega(t)$ $(0<t \leq 1)$ such that for some positive integer $N$ :

(a) $\mathrm{cl}[\Omega(t)] \subset \Omega\left(t^{\prime}\right)$ for $0<t<t^{\prime} \leq 1$.

(b) The complement $\mathbb{C} \backslash \Omega(t)$ consists of $N$ connectivity components for all $t$.

(c) The boundaries $\partial \Omega(t)$ are all $C^{2}$-smooth and vary in a $C^{2}$-smooth fashion with $t$.

(d) The intersection $\bigcap_{t} \Omega(t)$ - what might be called the skeleton - is a continuum which is either a point or the union of a finite number of $C^{2}$-smooth curves, and, in particular, has zero area.

To simplify the notation, we write $G_{t}$ and $P_{t}$ for the Green function and the Poisson kernel of $\Omega(t)$, and $\mathbf{G}_{t}$ and $\mathbf{P}_{t}$ for the associated operators, respectively.

In the setting of (a)-(d), the classical variational formula of Hadamard [5] (cf. also [9, 10]) tells us how the Green function $G_{t}$ varies with $t$. We prefer to state the expression in integral form:

$$
G_{t}(z, w)=\int_{0}^{t} \int_{\partial \Omega(\tau)} P_{\tau}(z, \zeta) P_{\tau}(w, \zeta) \varrho(\zeta) \mathrm{d} s(\zeta) \mathrm{d} \tau,
$$

where $\varrho(\zeta)$ is the rate at which the boundary $\partial \Omega(\tau)$ moves at $\zeta \in \partial \Omega(\tau)$ along the direction of the exterior unit normal vector as $\tau$ grows. Note that the index $\tau$ is uniquely determined by the condition $\zeta \in \partial \Omega(\tau)$. Also recall that the Poisson kernel is extended to equal 0 outside the closure of the domain where it was originally defined.

For our purposes it is important to note that, for each $t$, the boundaries $\partial \Omega(\tau)(0<\tau<t)$ essentially foliate the entire domain $\Omega(t)$ in the sense that their union (which is disjoint by assumption (a)) equals $\Omega(t)$ except for the skeleton. This makes it possible to compute area integrals over $\Omega(t)$ using the expression

$$
\int_{\Omega(t)} f \mathrm{~d} A=\int_{0}^{t}\left\{\int_{\partial \Omega(\tau)} f \varrho \mathrm{d} s\right\} \mathrm{d} \tau
$$

reminiscent of polar coordinates. It follows from (3.2) that $\varrho>0$ almost everywhere on $\Omega(1)$.

Remark 3.1. An explicit description of a setup of the above kind and a proof of Hadamard's formula in that context can be found in Schippers [10]. He assumes that the boundaries $\partial \Omega(t)$ are furnished by a collection of injective and non-overlapping $C^{2}$-smooth homotopies $F_{j}(t, \theta)$ $(j=1, \ldots, N)$ where $\theta \in[0,2 \pi]$ (with endpoints identified). Then, at each point $\zeta=F_{j}(t, \theta)$, a routine computation shows that $\varrho(\zeta)=\left|\operatorname{det} \mathrm{D} F_{j}(t, \theta)\right| /\left|\partial F_{j}(t, \theta) / \partial \theta\right|$ with $\mathrm{D} F_{j}$ denoting the Jacobian matrix. The expression (3.2) now follows by usual change of variables using the maps $F_{j}$.

3.2. The Hadamard operator. We keep the setting of (a)-(d) above, and for each $\zeta \in \Omega(1)$, except the points of the skeleton, let $\tau(\zeta)$ be the unique value of $t$ such that $\zeta \in \partial \Omega(t)$. In words, $\tau(\zeta)$ is the time when the front $\partial \Omega(t)$ passes the point $\zeta$. For each $0<t \leq 1$, we introduce the Hadamard operator $\mathbf{Q}_{t}$ by

$$
\mathbf{Q}_{t} f(z):=\int_{\Omega(t)} P_{\tau(\zeta)}(z, \zeta) f(\zeta) \mathrm{d} A(\zeta),
$$

whenever the integral makes sense. In terms of signals, $\mathbf{Q}_{t}$ maps the unit point mass at $\zeta \in \Omega(t)$ to the Poisson kernel function $z \mapsto P_{\tau(\zeta)}(z, \zeta)$, treated as zero for $z \in \mathbb{C} \backslash \Omega(\tau(\zeta))$. Note that $\mathbf{Q}_{t} f$ 
is always supported in $\mathrm{cl}[\Omega(t)]$ and $\mathbf{Q}_{t} f=\mathbf{Q}_{1}\left(1_{\Omega(t)} f\right)$ where $1_{\Omega(t)}$ stands for the characteristic function of $\Omega(t)$. Similarly, we define the adjoint Hadamard operator

$$
\mathbf{Q}_{t}^{*} f(\zeta):=1_{\Omega(t)}(\zeta) \int_{\Omega(t)} P_{\tau(\zeta)}(z, \zeta) f(z) \mathrm{d} A(z),
$$

whenever the integral makes sense.

We note the linear appearance of the Poisson kernel in the definition of the Hadamard operator, while it appears in a bilinear fashion in Hadamard's formula (3.1). These two objects are intimately related; in fact, as we will see in Section 5 . Hadamard's formula corresponds to the calculation of correlations for the stochastic field generated by the Hadamard operator.

We collect the main properties of $\mathbf{Q}_{t}$ and $\mathbf{Q}_{t}^{*}$ in the following proposition. We denote by $\Delta_{t}$ the Laplacian on $\Omega(t)$, and recall that the powers of $-\Delta_{t}$ are to be understood with respect to vanishing Dirichlet boundary data.

Proposition 3.2. The operators $\left[-\Delta_{t}\right]^{1 / 2} \mathbf{Q}_{t}$ and $\mathbf{Q}_{t}^{*}\left[-\Delta_{t}\right]^{1 / 2}$ are both unitary on $L^{2}(\Omega(t))$. Moreover, $\mathbf{Q}_{t} \mathbf{Q}_{t^{\prime}}^{*}=\left[-\Delta_{t \wedge t^{\prime}}\right]^{-1}$ for every $t$ and $t^{\prime}$, where $t \wedge t^{\prime}$ denotes the minimum of $t$ and $t^{\prime}$.

In view of identity (2.1), the proposition can be rephrased as saying that $\mathbf{Q}_{t}$ is an isometric isomorphism $L^{2}(\Omega(t)) \rightarrow H_{0}^{1}(\Omega(t))$ and $\mathbf{Q}_{t}^{*}$ is an isometric isomorphism $H^{-1}(\Omega(t)) \rightarrow L^{2}(\Omega(t))$, where $H^{-1}(\Omega(t))$ is the dual of $H_{0}^{1}(\Omega(t))$ under the duality pairing induced by the $L^{2}$ inner product.

The following observation, which will be needed in the proof of Proposition 3.2, is helpful in understanding the evolution of the operator $\mathbf{Q}_{t}$ as the domain $\Omega(t)$ varies.

Lemma 3.3. For $0<t<t^{\prime} \leq 1$ and $f \in L^{2}\left(\Omega\left(t^{\prime}\right)\right)$, the function

$$
\mathbf{Q}_{t^{\prime}} f(z)-\mathbf{Q}_{t} f(z)=\int_{\Omega\left(t^{\prime}\right) \backslash \Omega(t)} P_{\tau(\zeta)}(z, \zeta) f(\zeta) \mathrm{d} A(\zeta)
$$

equals $\mathbf{Q}_{t^{\prime}} f(z)$ on $\Omega\left(t^{\prime}\right) \backslash \Omega(t)$, and on $\Omega(t)$ it is harmonic and its boundary values on $\partial \Omega(t)$ are those of $\mathbf{Q}_{t^{\prime}} f$.

Proof. This follows from the easily verified fact that $\mathbf{Q}_{t} f$ vanishes in $\mathbb{C} \backslash \Omega(t)$, together with the observation that $z \mapsto P_{\tau(\zeta)}(z, \zeta)$ is harmonic in $\Omega(\tau(\zeta))$.

Proof of Proposition 3.2 We start by addressing the last assertion. A computation with a test function $f \in C_{0}^{\infty}(\mathbb{C})$ gives

$$
\begin{aligned}
\mathbf{Q}_{t} \mathbf{Q}_{t^{\prime}}^{*} f(z) & =\int_{\Omega\left(t^{\prime}\right)}\left\{\int_{\Omega(t)} 1_{\Omega\left(t^{\prime}\right)}(\zeta) P_{\tau(\zeta)}(z, \zeta) P_{\tau(\zeta)}(w, \zeta) \mathrm{d} A(\zeta)\right\} f(w) \mathrm{d} A(w) \\
& =\int_{\Omega\left(t^{\prime}\right)}\left\{\int_{0}^{t \wedge t^{\prime}} \int_{\partial \Omega(\tau)} P_{\tau}(z, \zeta) P_{\tau}(w, \zeta) \varrho(\zeta) \mathrm{d} s(\zeta) \mathrm{d} \tau\right\} f(w) \mathrm{d} A(w),
\end{aligned}
$$

using Fubini's theorem and the "polar coordinates" trick (3.2) with $\tau$ as the time variable. However, by Hadamard's formula (3.1), the integral in braces equals $G_{t \wedge t^{\prime}}(z, w)$. Consequently, $\mathbf{Q}_{t} \mathbf{Q}_{t^{\prime}}^{*} f(z)=\mathbf{G}_{t \wedge t^{\prime}} f(z)$, which gives $\mathbf{Q}_{t} \mathbf{Q}_{t^{\prime}}^{*}=\mathbf{G}_{t \wedge t^{\prime}} ;$ this is equivalent to the last assertion.

Turning to the operator $\mathbf{Q}_{t}^{*}\left[-\Delta_{t}\right]^{1 / 2}$, we note that for all $f, g \in C_{0}^{\infty}(\Omega(t))$ we get

$$
\left\langle\mathbf{Q}_{t}^{*}\left[-\Delta_{t}\right]^{1 / 2} f, \mathbf{Q}_{t}^{*}\left[-\Delta_{t}\right]^{1 / 2} g\right\rangle_{\Omega(t)}=\left\langle\left[-\Delta_{t}\right]^{1 / 2} \mathbf{Q}_{t} \mathbf{Q}_{t}^{*}\left[-\Delta_{t}\right]^{1 / 2} f, g\right\rangle_{\Omega(t)}=\langle f, g\rangle_{\Omega(t)}
$$

by what we have already obtained. Hence $\mathbf{Q}_{t}^{*}\left[-\Delta_{t}\right]^{1 / 2}$ defines an isometry from $L^{2}(\Omega(t))$ into itself, and its adjoint $\left[-\Delta_{t}\right]^{1 / 2} \mathbf{Q}_{t}$ is a contraction with dense range in $L^{2}(\Omega(t))$. To show that both operators are unitary, it suffices to verify that $\left[-\Delta_{t}\right]^{1 / 2} \mathbf{Q}_{t}$ is injective. 
So suppose that $\left[-\Delta_{t}\right]^{1 / 2} \mathbf{Q}_{t} f=0$ for some $f \in L^{2}(\Omega(t))$. Since $\mathbf{Q}_{t} f$ belongs to $H_{0}^{1}(\Omega(t))$, this implies that $\mathbf{Q}_{t} f=0$. Then if $0<\tau<t$ and we replace $\mathbf{Q}_{t} f$ with the function having the same values on $\partial \Omega(\tau)$ and being harmonic in $\Omega(\tau)$, we still have the zero function. Thus, by Lemma 3.3, we must have $\mathbf{Q}_{\tau} f=0$ for all $\tau$ with $0<\tau<t$. Then the derivative must also vanish (for a.e. $\tau$ with $0<\tau<t$ ):

$$
\frac{\partial}{\partial \tau} \mathbf{Q}_{\tau} f(z)=\int_{\partial \Omega(\tau)} P_{\tau}(z, \zeta) f(\zeta) \varrho(\zeta) \mathrm{d} s(\zeta)=0, \quad z \in \Omega(\tau) .
$$

But this is the harmonic extension of $f \varrho$, and it can vanish everywhere only if $f \varrho=0$ holds a.e. on $\partial \Omega(\tau)$. Since $\varrho>0$ a.e., we conclude that $f=0$ a.e. This proves that $\left[-\Delta_{t}\right]^{1 / 2} \mathbf{Q}_{t}$ is injective.

\section{BASIC RANDOM FIELDS}

We briefly recall the construction and some basic properties of the white noise and Gaussian free fields. We primarily adopt the viewpoint of Gaussian Hilbert spaces. See Janson's book [8] for an account of such spaces and Sheffield [11] for a general survey of the Gaussian free field.

Throughout this section we assume that $\Omega$ is a bounded domain in the complex plane with a $C^{2}$-smooth boundary.

4.1. The white noise field. The (real-valued) white noise field on $\Omega$ is given formally as a random linear combination

$$
\Phi=\sum_{j=1}^{+\infty} \xi_{j} \phi_{j}
$$

where the $\xi_{j}(j=1,2, \ldots)$ are all independent standard Gaussian random variables, and the functions $\phi_{j}(j=1,2, \ldots)$ form an orthonormal basis of $L^{2}(\Omega)$. At times, we may want to express this as $\Phi \in \mathrm{WN}(\Omega)$. The above series does not converge (almost surely) in $L^{2}(\Omega)$. However, in terms of the bilinear form $\langle\cdot, \cdot\rangle_{\Omega}$ (i.e., the $L^{2}$ inner product on $\Omega$ ), it makes sense to consider

$$
\langle f, \Phi\rangle_{\Omega}=\sum_{j=1}^{+\infty} \xi_{j}\left\langle f, \phi_{j}\right\rangle_{\Omega}
$$

since this series converges in mean square to a Gaussian variable with mean 0 and variance $\|f\|_{L^{2}(\Omega)}^{2}$ for each $f \in L^{2}(\Omega)$. Thus we may view the white noise field as a Gaussian Hilbert space formed by the jointly Gaussian variables $\langle f, \Phi\rangle_{\Omega}$ - one for each $f \in L^{2}(\Omega)$ - whose covariance structure is given by

$$
\mathbb{E}\left[\langle f, \Phi\rangle_{\Omega}\langle g, \Phi\rangle_{\Omega}\right]=\langle f, g\rangle_{\Omega}
$$

Here, the symbol $\mathbb{E}$ stands for the expectation operation. Note, in particular, that the law for the field is independent of the choice of basis - this is somewhat akin to the fact that the reproducing kernel of a Hilbert space of functions, which may be written in terms of an orthonormal basis, is independent of the choice of the basis (cf. [1]).

Alternatively, $\Phi$ can be understood as a random element in a space bigger than $L^{2}(\Omega)$. One way is to define $\Phi$ as a random distribution in the Sobolev space $H^{-1-\epsilon}(\Omega)$ for some $\epsilon>0$ such that its action on test functions $f \in C_{0}^{\infty}(\Omega)$ yields random variables $\langle f, \Phi\rangle_{\Omega}$ as described above. This is possible because the natural embedding of $L^{2}(\Omega)$ into $H^{-1-\epsilon}(\Omega)$ is a Hilbert-Schmidt operator; see [11, Sec. 2.2]. 
An important property of the white noise field $\Phi$ is the independent action of the "vibrations" in different parts of $\Omega$. For instance, if $\Omega=\Omega_{1} \cup \Omega_{2} \cup E$, where the union is mutually disjoint, and $\Omega_{j}$ is open for $j=1,2$, while $E$ has zero area, then

$$
L^{2}(\Omega)=L^{2}\left(\Omega_{1}\right) \oplus L^{2}\left(\Omega_{2}\right)
$$

as orthogonal subspaces in a Hilbert space. As a result, $\Phi=\Phi_{1}+\Phi_{2}=\Phi_{1} \boxplus \Phi_{2}$, where $\Phi_{1}$ is the field $\Phi$ conditioned to vanish on $\Omega_{2}$, and $\Phi_{2}$ is the field $\Phi$ conditioned to vanish on $\Omega_{1}$. Here, the symbol $\boxplus$ is used to indicate that the summands are independent random fields. In a natural sense, $\Phi_{j} \in \mathrm{WN}\left(\Omega_{j}\right)$ for $j=1,2$.

4.2. The Gaussian free field. The Gaussian free field on $\Omega$ with vanishing boundary data, which we will denote as $\Psi \in \operatorname{GFF}_{0}(\Omega)$, can be defined in a way that is analogous to the white noise field above. Instead of the space $L^{2}(\Omega)$, we just work with the Sobolev space $H_{0}^{1}(\Omega)$ (see Section 2.2). Then we obtain a Gaussian Hilbert space formed by centred Gaussian variables $\langle f, \Psi\rangle_{\Omega, \nabla}$ for $f \in H_{0}^{1}(\Omega)$ with the property that

$$
\mathbb{E}\left[\langle f, \Psi\rangle_{\Omega, \nabla}\langle g, \Psi\rangle_{\Omega, \nabla}\right]=\langle f, g\rangle_{\Omega, \nabla} .
$$

Again, $\Psi$ does not (almost surely) determine an element of $H_{0}^{1}(\Omega)$, yet it can be defined as a random element in the Sobolev space $H^{-\epsilon}(\Omega)$ for any $\epsilon>0$. In view of (2.1), the Gaussian free field may be retrieved from the white noise by $\Psi=[-\Delta]^{-1 / 2} \Phi$ in the sense of distribution theory. Moreover, the action of $\Psi$ on $L^{2}(\Omega)$ is given by $\langle f, \Psi\rangle_{\Omega}=\left\langle[-\Delta]^{-1} f, \Psi\right\rangle_{\Omega, \nabla}$, so that

$$
\mathbb{E}\left[\langle f, \Psi\rangle_{\Omega}\langle g, \Psi\rangle_{\Omega}\right]=\left\langle f,[-\Delta]^{-1} g\right\rangle_{\Omega}
$$

for $f, g \in L^{2}(\Omega)$. In fact, the definition of $\langle f, \Psi\rangle_{\Omega}$ can be extended to all $f \in H^{-1}(\Omega)$ since $[-\Delta]^{-1}$ maps $H^{-1}(\Omega)$ into $H_{0}^{1}(\Omega)$.

A main difference between the fields $\operatorname{GFF}_{0}(\Omega)$ and $\mathrm{WN}(\Omega)$ is that while $\mathrm{WN}(\Omega)$ is purely local, in $\mathrm{GFF}_{0}(\Omega)$ we have non-trivial long-range correlations, due to the non-local nature of the operator $[-\Delta]^{-1 / 2}$.

\section{The Gaussian free field via the Hadamard operator}

Throughout this section, we work in the setting of the assumptions (a)-(d) described in Section 3.1. For brevity, we write $\Omega=\Omega(1)$ for the largest domain under consideration.

5.1. Construction. We start by invoking the Hadamard operator to produce the Gaussian free field from the white noise field. We agree to write $\Psi_{0}=0$.

Theorem 5.1. Let $\Phi \in \mathrm{WN}(\Omega)$. For $0<t \leq 1$, let $\Psi_{t}:=\mathbf{Q}_{t} \Phi$, i.e.

$$
\left\langle f, \Psi_{t}\right\rangle_{\Omega}:=\left\langle\mathbf{Q}_{t}^{*} f, \Phi\right\rangle_{\Omega}, \quad f \in H^{-1}(\Omega) .
$$

Then $\Psi_{t} \in \operatorname{GFF}_{0}(\Omega(t))$. Moreover, the process $\Psi_{t}(0 \leq t \leq 1)$ has independent increments: for all $0=t(0)<\cdots<t(n)=1$ and $f_{1}, \ldots, f_{n} \in H^{-1}(\Omega)$, the random variables $\left\langle f_{j}, \Psi_{t(j)}-\Psi_{t(j-1)}\right\rangle_{\Omega}$ $(j=1, \ldots, n)$ are independent.

Proof. For $0<t, t^{\prime} \leq 1$ and $f, g \in H^{-1}(\Omega)$, we have from the definitions that

$$
\mathbb{E}\left[\left\langle f, \Psi_{t}\right\rangle_{\Omega}\left\langle g, \Psi_{t^{\prime}}\right\rangle_{\Omega}\right]=\mathbb{E}\left[\left\langle\mathbf{Q}_{t}^{*} f, \Phi\right\rangle_{\Omega}\left\langle\mathbf{Q}_{t^{\prime}}^{*} g, \Phi\right\rangle_{\Omega}\right]=\left\langle\mathbf{Q}_{t}^{*} f, \mathbf{Q}_{t^{\prime}}^{*} g\right\rangle_{\Omega}=\left\langle f, \mathbf{Q}_{t} \mathbf{Q}_{t^{\prime}}^{*} g\right\rangle_{\Omega} .
$$

According to Proposition 3.2, $\mathbf{Q}_{t} \mathbf{Q}_{t^{\prime}}^{*}=\left[-\Delta_{t \wedge t^{\prime}}\right]^{-1}$. Taking $t=t^{\prime}$ we see that $\Psi_{t}$ satisfies the correlations condition (4.1) on $\Omega(t)$. Moreover, it follows easily that the increments of $\Psi_{t}$ are uncorrelated and hence, being jointly Gaussian, independent. 
Since $\left[-\Delta_{t}\right]^{-1}=\mathbf{G}_{t}$ (see Sec. 2.1), the covariance structure of the process $\Psi_{t}$ can be written in the form

$$
\begin{aligned}
\mathbb{E}\left[\left\langle f, \Psi_{t}\right\rangle_{\Omega}\left\langle g, \Psi_{t^{\prime}}\right\rangle_{\Omega}\right] & =\int_{\Omega} \int_{\Omega} G_{t \wedge t^{\prime}}(z, w) f(z) g(w) \mathrm{d} A(z) \mathrm{d} A(w) \\
& =\int_{\Omega} \nabla \mathbf{G}_{t \wedge t^{\prime}} f \cdot \nabla \mathbf{G}_{t \wedge t^{\prime}} g \mathrm{~d} A .
\end{aligned}
$$

In particular, the variance of $\left\langle f, \Psi_{t}\right\rangle_{\Omega}$ equals the energy integral $\int_{\Omega}\left|\nabla \mathbf{G}_{t} f\right|^{2} \mathrm{~d} A$.

In view of the definition of the Hadamard operator, we see from Theorem 5.1 that the Gaussian free field $\operatorname{GFF}_{0}(\Omega(t))$ can be thought of as obtained by integrating up (in terms of the area integral) the harmonic fields induced by Poisson extensions of point oscillations at each point $\zeta \in \partial \Omega(\tau)$ with $0<\tau<t$. Moreover, for $0<t<t^{\prime} \leq 1$ the $\mathrm{GFF}_{0}$ field on $\Omega\left(t^{\prime}\right)$ can be decomposed into independent fields:

$$
\Psi_{t^{\prime}}=\Psi_{t} \boxplus\left(\Psi_{t^{\prime}}-\Psi_{t}\right)=\mathbf{Q}_{t} \Phi \boxplus\left(\mathbf{Q}_{t^{\prime}}-\mathbf{Q}_{t}\right) \Phi .
$$

Here $\Psi_{t}$ is a GFF $F_{0}$ on the smaller domain $\Omega(t)$ with zero continuation to $\Omega\left(t^{\prime}\right) \backslash \mathrm{cl}[\Omega(t)]$, and $\Psi_{t^{\prime}}-\Psi_{t}$ is a field which is harmonic in $\Omega(t)$ and coincides with $\Psi_{t^{\prime}}$ on $\Omega\left(t^{\prime}\right) \backslash \mathrm{cl}[\Omega(t)]$ (see Lemma 3.3). The decomposition (5.2) expresses the Markov property of the Gaussian free field (cf. [11]).

5.2. Time-derivative of $\Psi_{t}$. Looking the increments at the infinitesimal scale, we may examine the time-derivative of the process $\Psi_{t}$. To this end, it is convenient to use the harmonic sweep operator $\mathbf{P}_{t}^{*}$ given by

$$
\mathbf{P}_{t}^{*} f(\zeta):=\int_{\Omega(t)} P_{t}(z, \zeta) f(z) \mathrm{d} A(z), \quad \zeta \in \partial \Omega(t)
$$

It is the adjoint of the Poisson extension operator. We see from the definition of $\mathbf{Q}_{t}^{*}$ that $\mathbf{Q}_{t}^{*} f(\zeta)=\mathbf{P}_{\tau}^{*} f(\zeta)$ provided that $\zeta \in \partial \Omega(\tau)$ with $0<\tau<t$. Consequently, for all $0<t, t^{\prime} \leq 1$ and $f, g \in L^{2}(\Omega)$, we have, in view of (3.2), that

$$
\mathbb{E}\left[\left\langle f, \Psi_{t}\right\rangle_{\Omega}\left\langle g, \Psi_{t^{\prime}}\right\rangle_{\Omega}\right]=\left\langle\mathbf{Q}_{t}^{*} f, \mathbf{Q}_{t^{\prime}}^{*}\right\rangle_{\Omega}=\int_{0}^{t \wedge t^{\prime}}\left\{\int_{\partial \Omega(\tau)} \mathbf{P}_{\tau}^{*} f \mathbf{P}_{\tau}^{*} g \varrho \mathrm{d} s\right\} \mathrm{d} \tau .
$$

Writing $\dot{\Psi}_{t}$ for the time-derivative of $\Psi_{t}$ in the sense of distribution theory, we get the expression

$$
\mathbb{E}\left[\left\langle f, \dot{\Psi}_{t}\right\rangle_{\Omega}\left\langle g, \dot{\Psi}_{t^{\prime}}\right\rangle_{\Omega}\right]=\delta_{0}\left(t-t^{\prime}\right) \int_{\partial \Omega(t)} \mathbf{P}_{t}^{*} f \mathbf{P}_{t}^{*} g \varrho \mathrm{d} s .
$$

Next, suppose that $\Xi_{t}$ is a weighted white noise process on $\partial \Omega(t)$ with weight $\varrho^{1 / 2}$, i.e., a Gaussian random field acting on $L^{2}(\partial \Omega(t))$ with correlation structure

$$
\mathbb{E}\left[\left\langle\phi, \Xi_{t}\right\rangle_{\partial \Omega(t)}\left\langle\psi, \Xi_{t}\right\rangle_{\partial \Omega(t)}\right]=\int_{\partial \Omega(t)} \phi \psi \varrho \mathrm{d} s
$$

for $\phi, \psi \in L^{2}(\partial \Omega(t))$. Let $\mathbf{P}_{t} \Xi_{t}$ be the Poisson extension of $\Xi_{t}$, i.e., the harmonic field on $\Omega(t)$ given by $\left\langle f, \mathbf{P}_{t} \Xi_{t}\right\rangle_{\Omega(t)}=\left\langle\mathbf{P}_{t}^{*} f, \Xi_{t}\right\rangle_{\partial \Omega(t)}$ for $f \in L^{2}(\Omega(t))$; in view of (5.4), it has the correlation structure

$$
\mathbb{E}\left[\left\langle f, \mathbf{P}_{t} \Xi_{t}\right\rangle_{\Omega(t)}\left\langle g, \mathbf{P}_{t} \Xi_{t}\right\rangle_{\Omega(t)}\right]=\int_{\partial \Omega(t)} \mathbf{P}_{t}^{*} f \mathbf{P}_{t}^{*} g \varrho \mathrm{d} s,
$$

which we compare with (5.3). We arrive at the following conclusions, whose detailed verification is left to the reader: 
Proposition 5.2. Suppose that $\Psi_{t}(0<t \leq 1)$ are given by Theorem 5.1 Then the following hold:

(a) For a fixed $f$, we have

$$
\left\langle f, \Psi_{t}\right\rangle_{\Omega} \stackrel{d}{=} \int_{0}^{t} \sqrt{\kappa(\tau)} d B(\tau) \stackrel{d}{=} B\left(\int_{0}^{t} \kappa(\tau) d \tau\right), \quad 0 \leq t \leq 1,
$$

where $B$ is a standard Brownian motion with $B(0)=0$ and $\kappa(\tau):=\int_{\partial \Omega(\tau)}\left|\mathbf{P}_{\tau}^{*} f\right|^{2} \varrho \mathrm{d}$ s. Here, " $\stackrel{d}{=}$ indicates that the processes have the same law (i.e., are versions of each other).

(b) The covariance structure of the differentiated process $\dot{\Psi}_{t}$ is induced by the process $\mathbf{P}_{t} \Xi_{t}$ :

$$
\mathbb{E}\left[\left\langle f, \dot{\Psi}_{t}\right\rangle_{\Omega}\left\langle g, \dot{\Psi}_{t^{\prime}}\right\rangle_{\Omega}\right]=\delta_{0}\left(t-t^{\prime}\right) \mathbb{E}\left[\left\langle f, \mathbf{P}_{t} \Xi_{t}\right\rangle_{\Omega(t)}\left\langle g, \mathbf{P}_{t} \Xi_{t}\right\rangle_{\Omega(t)}\right] .
$$

This identity should be understood in the sense of distribution theory.

Thus, in a sense, we are dealing with a time-changed Brownian motion in the time variable $t$. On the other hand, the individual independent increments (at infinitesimal scale) correspond to the harmonic extension to $\Omega(t)$ of weighted white noise fields along the boundaries $\partial \Omega(t)$.

Remark 5.3. The independent decomposition of the Gaussian free field given by Proposition 5.1 - and the continuous limit of that decomposition alluded to above - are in many ways analogous to the Brownian exploration of the Gaussian free along a space-filling curve, as in [11].

5.3. Boundary averages. It is rather well known (see e.g. [11, 2]) that the averages of the Gaussian free field on concentric circles follow a time-changed Brownian motion. We want to formulate an analogue of this fact for the averages of $\Psi_{1}$ on the associated flow of the boundaries $\partial \Omega(t)$.

We first note that Lemma 3.3 implies that for $f \in L^{2}(\Omega)$ and $0<t<1$, we have $\mathbf{P}_{t} \mathbf{Q}_{1} f=$ $\left(\mathbf{Q}_{1}-\mathbf{Q}_{t}\right) f$ on $\Omega(t)$. Passing to the adjoints, we get $\mathbf{Q}_{1}^{*} \mathbf{P}_{t}^{*} f=\left(\mathbf{Q}_{1}^{*}-\mathbf{Q}_{t}^{*}\right) f$ for functions $f$ on $\Omega(t)$. Thus we may evaluate $\Psi_{1}$ with the distribution $\mathbf{P}_{t}^{*} f$ ds as a "test function" to obtain

$$
\int_{\partial \Omega(t)} \Psi_{1} \mathbf{P}_{t}^{*} f \mathrm{~d} s=\left\langle\mathbf{P}_{t}^{*} f, \Psi_{1}\right\rangle_{\partial \Omega(t)}=\left\langle\mathbf{Q}_{1}^{*} \mathbf{P}_{t}^{*} f, \Phi\right\rangle_{\Omega}=\left\langle\left(\mathbf{Q}_{1}^{*}-\mathbf{Q}_{t}^{*}\right) f, \Phi\right\rangle_{\Omega}=\left\langle f, \Psi_{1}-\Psi_{t}\right\rangle_{\Omega} .
$$

More generally, this reasoning can be extended to the case where $f$ is replaced by a distribution whose support is contained in $\Omega(t)$. Then the following result is obtained.

Corollary 5.4. Let $X_{t}(f):=\int_{\partial \Omega(t)} \Psi_{1} \mathbf{P}_{t}^{*} f \mathrm{~d}$ sfor $0<t<1$ and distributions $f$ supported on the skeleton $\bigcap_{t} \Omega(t)$. Then, for all such distributions $f$ and $g$, and $0<t, t^{\prime}<1$, we have

$$
\mathbb{E}\left[X_{t}(f) X_{t^{\prime}}(g)\right]=\int_{t \vee t^{\prime}}^{1}\left\{\int_{\partial \Omega(\tau)} \mathbf{P}_{\tau}^{*} f \mathbf{P}_{\tau}^{*} g \varrho \mathrm{d} s\right\} \mathrm{d} \tau,
$$

where $t \vee t^{\prime}$ is the maximum of $t$ and $t^{\prime}$. In particular, $X_{t}(f) \stackrel{d}{=} B\left(\int_{t}^{1} \kappa(\tau) d \tau\right)$ with $\kappa(\tau)$ defined as in Proposition 5.2

Thus, the process $X_{t}(f)(0<t<1)$ - the values of which are the integrals along the curves $\partial \Omega(t)$ of the field $\Psi_{1} \in \operatorname{GFF}_{0}(\Omega)$ with respect to the (signed) measures $\mathbf{P}_{t}^{*} f \mathrm{~d} s$ - obeys the law of a time-reversed Brownian motion running at a variable speed.

For instance, if $\Omega(t)$ is the open disc of radius $t$ centred at the origin and $f=\delta_{0}$, the unit point mass at 0 , we have $\varrho \equiv 1$ and $\mathbf{P}_{t}^{*} \delta_{0} \equiv(2 \pi t)^{-1}$. Then $\kappa(t)=(2 \pi t)^{-1}$, and we obtain the well-known result that the average of $\mathrm{GFF}_{0}$ on concentric circles of radii $e^{-2 \pi t}$ has exactly the law of a Brownian motion. 
Remark 5.5. It is possible to supply an expression of type (5.1) for the covariance $\mathbb{E}\left[X_{t}(f) X_{t^{\prime}}(g)\right]$ altough it is slightly more delicate. We first observe that the Green potential $\mathbf{G}_{1} f$ is well defined (as a distribution) for distributions $f$ supported in the skeleton, and that $\mathbf{G}_{1} f$ is harmonic in $\Omega$ off the skeleton and vanishes on $\partial \Omega$. We let $\tilde{\mathbf{G}}_{t} f$ denote the function which equals $\mathbf{G}_{1} f$ on $\Omega \backslash \Omega(t)$, and is harmonically extended from its boundary values to $\Omega(t)$. Then

$$
\mathbb{E}\left[X_{t}(f) X_{t^{\prime}}(g)\right]=\int_{\Omega} \nabla \tilde{\mathbf{G}}_{t \vee t^{\prime}} f \cdot \nabla \tilde{\mathbf{G}}_{t \vee t^{\prime}} g \mathrm{~d} A .
$$

Remark 5.6. (a) As we have seen, the $\mathrm{GFF}_{0}$ field can be derived from the white noise field by applying the inverse of the square root of minus the Laplacian to $\mathrm{WN}$. The conformal invariance of the Laplacian leads to the corresponding conformal invariance of $\mathrm{GFF}_{0}$. If we replace the Laplacian by $\operatorname{div} \frac{1}{\omega} \nabla$ (which appears, e.g., in Calderón's inverse conductivity problem), the process retains the conformal invariance structure if we allow the weight $\omega$ to change correspondingly. Here, the weight function $\omega$ may be assumed positive and smooth. It should be possible to extend the use the Hadamard variation technique to this more general setting. We might also want to consider in the complex-valued setting the operators introduced by Garabedian [4].

(b) Hadamard's variation formula also applies in the setting of the biharmonic equation with vanishing Dirichlet data (see [5] for the original result, and [6, 7] for the integral version). This should lead to a corresponding Hadamard operator in this setting, and that should have applications to the associated random fields.

Acknowledgements. The authors thank Michael Benedicks and Kalle Kytölä for their interest in this work, and Boualem Djehiche for help with references. The first author also thanks Stanislav Smirnov for the inspiring conference "Conformal maps from probability to physics" at Monte Verità, Ascona, in 2010, and Pavel Wiegmann for several interesting conversations. The second author acknowledges the hospitality of the Department of Mathematics at the Royal Institute of Technology in Stockholm during his visit in 2011.

\section{REFERENCES}

[1] Aronszajn, N., Theory of reproducing kernels. Trans. Amer. Math. Soc. 68 (1950), 337-404.

[2] Duplantier, B., Sheffield, S., Liouville quantum gravity and KPZ. Invent. Math. 185 (2011), no. 2, 333-393.

[3] Evans, L., Partial Differential Equations, 2nd ed. Amer. Math. Soc., Providence, 2010.

[4] Garabedian, P. R., A partial differential equation arising in conformal mapping. Pacific J. Math. 1 (1951), 485-524.

[5] Hadamard, J., Mémoire sur le problème d'analyse relatifà l'équilibre des plaques élastiques encastrées. Mémoires présentés par divers savants à l'Académie des Sciences 33 (1908).

[6] Hedenmalm, H., A computation of Green functions for the weighted biharmonic operators $\Delta|z|^{-2 \alpha} \Delta$, with $\alpha>-1$. Duke Math. J. 75 (1994), no. 1, 51-78.

[7] Hedenmalm, H., Jakobsson, S., Shimorin, S., A biharmonic maximum principle for hyperbolic surfaces. J. Reine Angew. Math. 550 (2002), 25-75.

[8] Janson, S., Gaussian Hilbert spaces. Cambridge Univ. Press, Cambridge, 1997.

[9] Nehari, Z., Conformal mapping. McGraw-Hill, New York, 1952.

[10] Schippers, E., Behaviour of kernel functions under homotopic variations of planar domains. Comput. Methods Funct. Theory 4 (2004), no. 2, 283-298.

[11] Sheffield, S., Gaussian free field for mathematicians. Probab. Theory Related Fields 139 (2007), no. 3-4, 521-541.

Hedenmalm: Department of Mathematics, KTH Royal Institute of Technology, S-10044 Stockholm, Sweden

E-mail address: haakanh@math.kth.se

Nieminen: Department of Mathematics and Statistics, University of Helsinki, Box 68, FI-00014 Helsinki, FINLAND

E-mail address: pjnieminacc.helsinki.fi 